\title{
Paisajes en transición: gradientes urbano-rurales y antropización del bosque templado andino del sur de Chile
}

\author{
Gonzalo Vergara', ${ }^{1}$ y José Tomás Ibarra $3,4,5$
}

\begin{abstract}
RESUMEN
La asignación de categorías espaciales arbitrarias en los análisis de paisaje es un factor limitante al interpretar procesos socio-ecológicos que ocurren de forma continua en el paisaje. La noción de gradiente urbano-rural se ha utilizado para sortear dicho límite. En este trabajo analizamos la relación entre la estructura de los parches de vegetación nativa y el grado de antropización del paisaje en una cuenca del sur de Chile, con relación a la distancia a la ciudad. Se utilizaron 14 transectos para testear la disposición en gradientes de la antropización utilizando el Índice de Hemerobia, métricas de paisaje y topografía. Los resultados presentaron gradientes de intervención antrópica y de dominancia de clases en base a la distancia del centro urbano. Se distinguen tres secciones asociadas a una dominancia del área urbana, un sector de transición y de la clase bosque. Las transiciones del gradiente son la base para la vinculación entre patrones espaciales, procesos socio-ecológicos e intervención antrópica.
\end{abstract}

Palabras clave: Ecología del Paisaje, Índice de Hemerobia, Métricas de paisaje, Patrones espaciales, Sistemas socio-ecológicos.

\begin{abstract}
The assignment of arbitrary spatial categories in landscape analysis is a limiting factor for interpreting socio-ecological processes that occur continuously in the landscape. The notion of urban-rural gradients has been used for avoiding this limitation. In this paper we analyze the relation between the structure of native vegetation patches and the degree of landscape anthropization in a watershed of southern Chile, in relation to the distance to the city. We used 14 transects to test the arrangement on anthropization gradients using the Hemeroby Index, landscape metrics, and topography. We found gradients of both anthropogenic intervention and class dominance in relation to the distance to the urban center. We distinguished three major sections associated with a dominance of the urban area, the forest class, and a transition zone. The transitions in the gradient are the basis for linking spatial patterns, socio-ecological processes and anthropogenic influences.
\end{abstract}

Keywords: Landscape Ecology, Hemeroby Index, Landscape metrics, Spatial patterns, Socio-ecological systems.

\footnotetext{
Heidelberg Center Lateinamerika, Universität Heidelberg. Santiago, Chile. E-mail: gvergara@heidelbergcenter.cl

Centro de Estudio y Conservación del Patrimonio Natural (CECPAN). Ancud, Chile.

Laboratorio ECOS (Ecología-Complejidad-Sociedad), Centro de Desarrollo Local, Educación e Interculturalidad (CEDEL), Pontificia Universidad Católica de Chile. Villarrica, Chile. E-mail: jtibarra@uc.cl

Centro de Estudios Interculturales e Indígenas (CIIR), Facultad de Ciencias Sociales, Pontificia Universidad Católica de Chile. Santiago, Chile.

Núcleo Milenio Centro para el Impacto Socioeconómico de las Políticas Ambientales (CESIEP) y Centro de Ecología Aplicada y Sustentabilidad (CAPES) Pontificia Universidad Católica de Chile. Santiago, Chile.
} 
Las acepciones que se manejan sobre el concepto "paisaje" pueden ser diversas, abarcando dimensiones tales como la estética, ecológica, cultural e interpretativa (Tesser 2000). Entre las diversas posibilidades de análisis que ofrece la polisemia del "paisaje", se encuentra la Ecología del Paisaje. En la Ecología del Paisaje se "analizan las características estructurales y morfológicas que componen un territorio en momento determinado y/o su evolución a lo largo del tiempo, infiriendo a la vez en su incidencia a nivel de funcionalidad ecológica" (Vila et al. 2006, p. 155). En la práctica, y al componerse de la Geografía y la Ecología, la Ecología del Paisaje permite evaluar problemáticas de uso y cobertura de suelo, para luego sugerir directrices de ordenación del espacio geográfico a diversas escalas.

La Ecología del Paisaje permite asimilar porciones de territorio que comparten cierto grado de similitud, generando unidades homogéneas a partir de los elementos que componen el paisaje en su integridad (Serrano Giné 2012). La base para el análisis de la dimensión de las relaciones espaciales, entre unidades homogéneas, se construye a partir de modelos de cobertura y uso de suelo. Estos modelos definen la estructura del paisaje, la base de los elementos que componen un paisaje, a partir de una evaluación restringida que reduce la realidad a límites claros y precisos. Cushman, Gutzweiler, Evans y McGarigal plantean que, durante el análisis de variables en categorías discretas "la información cualitativa acerca de cómo las variables varían en el espacio y tiempo se pierde, dejando parches rígidos e internamente homogéneos" (2010, p. 84). A partir de esto, los autores identifican cuatro principales problemas al trabajar con categorías discretas en el uso o cobertura de suelo: 1) la decisión subjetiva de cómo caracterizar y definir límites restringirá la inferencia de las relaciones entre distintas coberturas de suelo; 2) los límites de parches basados en criterios del observador pueden diferir de criterios ecosistémicos; 3 ) cuando los parches están creados, la variabilidad interna se elimina dentro de los parches de la misma categoría, reduciendo las posibilidades a diferencias entre categorías; y 4) se asume homogeneidad en regiones de atributos simples o compuestos (Cushman et al. 2010).

La Ecología del Paisaje no está exenta de limitaciones o desafíos. Al considerar microunidades de paisaje dentro de la totalidad del paisaje considerado como área de estudio, es posible reconocer la diversidad de elementos estructurales y procesos que ocurren simultáneamente, tales como la dominancia de distintas clases en función de la distancia al centro urbano (Qureshi, Haase y Coles 2014). Es dentro de estas posibilidades en donde los análisis cuantitativos dentro de la Ecología del Paisaje se ven sesgados por la definición de unidad de paisaje y la valoración discreta de la unidad, donde el "paradigma del gradiente" supone un avance hacia la identificación de procesos espaciales (Cushman et al. 2010). Esta postura respecto a los procesos, abarcando la estructura del paisaje, supone una mirada a las intervenciones en múltiples dimensiones: ecológicas, sociales, económicas y políticas (Torres-Gómez et al. 2009). En términos prácticos, y al igual que muchas otras disciplinas científicas, la Ecología del Paisaje debe hacerse camino hacia la integración en la planificación informada y no sólo desde una perspectiva descriptiva o analítica de alta complejidad, además de la constante búsqueda de la transdisciplinariedad, inherente a su origen (Arroyo-Rodríguez, Moreno y Galán-Acedo 2017). Arroyo-Rodríguez, Moreno y Galán-Acedo (2017) identifican que la mayoría de los estudios en Ecología del Paisaje se concentran en la perspectiva animal y vegetal, dejando como foco secundario las intervenciones antrópicas y otros procesos socio-ecológicos. De esta manera, la Ecología del Paisaje debiera favorecer la identificación y gestión de recursos para la planificación de usos de suelo y enfatizando la relevancia de coberturas clave para la mantención de procesos ecosistémicos, tal como la planificación de 
territorios con un alto porcentaje de coberturas de suelo de interés para la conservación por motivos ecosistémicos, estéticos, culturales y económicos (Flesch y Steidl 2010; Ibarra et al. 2012).

Los paisajes, dentro de su amplia gama de acepciones, son el resultado de la modelación a partir de procesos socio-ecológicos (Tenerelli, Demšar y Luque 2016). La característica fundamental de estos procesos es que las relaciones espaciales no se desarrollan dentro de límites claros y con restricciones cualitativas otorgadas arbitrariamente, tal como se observa en los modelos de cobertura y uso de suelo, sino con gradientes altamente variables según el contexto (Cushman et al. 2010). Un claro ejemplo corresponde a las múltiples interpretaciones de los límites de la ciudad, ¿sus límites se concentran en la extensión superficial del área urbana dada por la cobertura de suelo o sobre las relaciones que tiene con su entorno inmediato? De esta manera, se puede encontrar una amplia gama de insumos que varían en complejidad para ser incorporados en un Sistema de Información Geográfica (SIG), desde insumos continuos simples, tales como la temperatura y la altitud, hasta insumos de mayor complejidad como el grado de antropización.

\section{Intervenciones antrópicas en el paisaje: hacia el análisis de gradientes espaciales}

La antropización del paisaje, como manera de representación continua en el espacio, se analizó por vez primera mediante el gradiente urbano-rural (McDonnell y Pickett 1990). En este contexto, la antropización urbano-rural se entendió como la disminución del proceso de urbanización desde la zona urbana a la zona rural, generando un gradiente espacial de influencia antrópica (Medley et al., 1995). Posteriormente, Steinhardt et al. (1999) proponen el Índice de Hemerobia (IH) para evaluar las intervenciones humanas en los ecosistemas. Este índice permite cuantificar el nivel de intervención en base a los diferentes tipos de cobertura de suelo que existen en un determinado paisaje. La posibilidad de tratar unidades dentro de transectos permite analizar este paisaje de manera continua y sorteando límites discretos y arbitrarios. Más aún, este índice permite relacionar la intervención antrópica en gradientes urbano-rurales desde un área urbana presente en diversos escenarios de complejidad topográfica y natural. Finalmente, el análisis del IH sobre un gradiente urbano-rural permite evaluar las relaciones espaciales de intervención antrópica desde un centro urbano hacia el exterior y con una multiplicidad de factores socio-ecológicos asociados (Walz y Stein 2014).

Dentro de los factores que condicionan la intervención de las áreas urbanas sobre el paisaje se encuentra la topografía, a través de la resistencia que supone ésta sobre el crecimiento del área urbana y sus implicancias directas respecto a la planificación territorial (Colantoni et al. 2016). Una de las consecuencias inmediatas de la topografía corresponde a la geometría semi-regular de las áreas urbanas presentes en extensiones llanas mientras que, en una topografía accidentada, la geometría debe adaptarse a ella. Este último caso se presenta en las áreas urbanas de la zona lacustre inserto en los paisajes templados del sur de Chile, donde el intrincado relieve es el resultado del constante modelado volcánico, el modelado glacial previo y el modelado fluvial actual (Gedda 2011; Vergara 2013). Estos paisajes se caracterizan por sus marcados gradientes altitudinales de formaciones y pisos vegetacionales que forman parte del ecosistema de bosque templado andino (Gedda 2011). La complejidad espacial de la cuenca del lago Villarrica $\left(39^{\circ} \mathrm{S}\right.$, $71^{\circ} \mathrm{O}$ ) es un ejemplo de cómo se condicionan los procesos socio-ecológicos a la topografía. Por 
una parte, las áreas urbanas (Pucón y Villarrica) se sitúan en las áreas llanas disponibles alrededor del lago, mientras que las actividades económicas predominantes parecieran buscar adaptarse al modelado terrestre.

El objetivo de esta investigación fue analizar la relación entre la estructura de los parches de vegetación nativa y el grado de antropización del paisaje en la cuenca del Lago Villarrica, sur de Chile, estableciendo si la pérdida de vegetación en el gradiente urbano-rural se relaciona con la distancia a la ciudad de Pucón. A partir de esta relación entre intervención antrópica y la estructura del paisaje, se espera (i) la existencia de un gradiente urbano-rural con una tendencia de disminución del grado de antropización del paisaje al alejarse del centro urbano y (ii) una disminución de la diversidad del paisaje al alejarse del centro urbano. Para testear estas hipótesis, se utilizó el Índice de Hemerobia, métricas de paisaje y topografía, los cuales permitieron evaluar los gradientes urbano-rurales y las transiciones espaciales de uso del paisaje en una cuenca topográficamente compleja y sujeta a distintas presiones de uso del territorio en el sur de Chile. Es importante destacar que este tipo de estudios no han sido desarrollados ampliamente en Chile, encontrándose los estudios de Torres-Gómez et al. (2009) en Coyhaique, de la Barrera (2012) y Picon, de la Barrera y Ruiz (2016) en Santiago.

\section{Características y relevancia del área de estudio}

La cuenca del Lago Villarrica está ubicada en la región central lacustre y del llano glacio-volcánico en la Región de La Araucanía, Chile (Figura 1), abarcando las comunas de Pucón y Curarrehue, además de una sección de Villarrica y Cunco. La topografía se modela en terrenos cordilleranos con volcanismo activo y sobre una morfología de colinajes aislados, predominando el cordón volcánico Villarrica-Quetrupillán-Lanín y los lagos Villarrica y Caburgua (Gedda 2011). La irregularidad de la topografía condiciona el trazado de los cursos de agua a través de la forma que muestran las numerosas cuencas tributarias y en la marcada sinuosidad de los lechos de ríos. Con una pendiente media de $17 \%$, la localización de las actividades humanas se concentra en zonas de baja pendiente y a orillas del lago Villarrica (Vergara 2013), con la ciudad de Villarrica al oeste y la ciudad de Pucón al este.

La cuenca presenta ecosistemas de relevancia socio-ecológica, entre ellos humedales y bosques nativos, clasificados como sistemas de alta fragilidad al estar sujetos a la evolución y ocurrencia de procesos eruptivos (Costantini et al. 2012). Con un total de 287.921 ha, la cuenca dispone de 88.540 ha (31\%) dentro del Sistema Nacional de Áreas Silvestres Protegidas del Estado (SNASPE) y 2.388 ha (1\%) en Áreas Protegidas Privadas (APPs) (en base a cálculos propios; Figura 1). Las secciones de estas áreas protegidas se encuentran principalmente en sectores altos, cubriendo extensiones de bosque nativo antiguo sobre los $700 \mathrm{~m}$ de altitud (Caviedes y Ibarra 2017; Ibarra et al. 2017). Tanto dentro como fuera del conjunto de áreas protegidas, destacan distintas especies arbóreas, las que incluyen a representantes de los géneros Nothofagus, Laurelia, Gevuina, Persea, Saxegothaea, Laureliopsis y Araucaria, entre otros (Caviedes y Ibarra 2017; Vergara 2013). Se ha sugerido que la mantención de la vegetación nativa en zonas altas estaría asociada, entre otros factores, a las características topográficas del paisaje que determinan un sistema de difícil acceso a las actividades humanas intensivas (Petitpas et al. 2016). 
Figura 1

Cuenca del lago Villarrica en la zona andina de la Región de La Araucanía, Chile

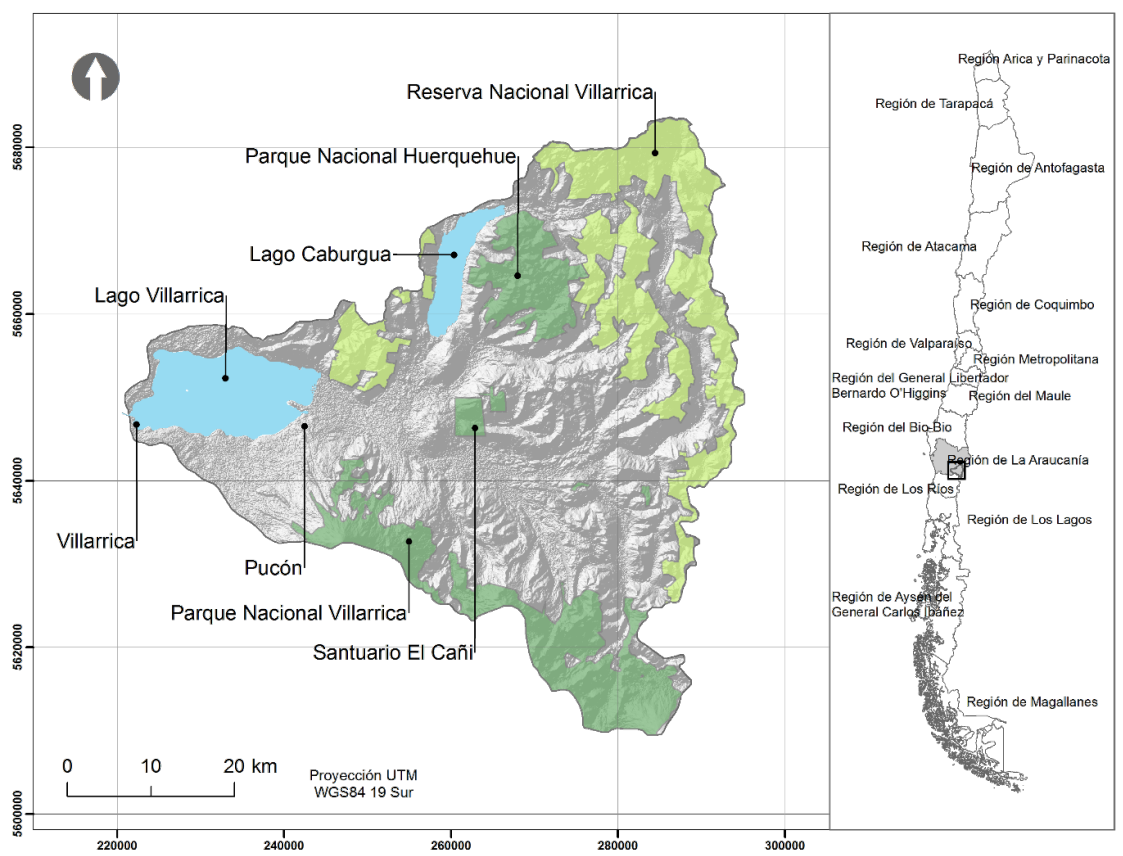

Fuente: Elaboración propia.

Dada la calidad estética y la alta afluencia de turistas en periodo estival, la funcionalidad del paisaje, desde una perspectiva socio-ecológica, se ha modificado fuertemente durante las últimas dos décadas, definiendo un fuerte crecimiento de la industria del turismo (Martínez y Terra 2012). Las actividades propias del turismo han generado una tensión respecto a la dinámica socioeconómica que se ha plasmado directamente en el modelo de planificación territorial de la ciudad de Pucón (Espinosa 2016). Si bien en el área urbana se concentra la modificación directa del uso y cobertura de suelo, principalmente sobre el aumento del área urbana (Mao, Meng y Wang 2014), la literatura indica cambios socio-ecológicos adicionales, tales como el aumento de la presión sobre recursos básicos como el agua (Zu-Yong, Ju-Qin y Fu-Hua 2013), la alteración de procesos ecológicos en la transición desde y hacia áreas protegidas (Ballantyne y Pickering 2015; Ibarra y Martin 2015; Petitpas et al. 2016; Ibarra et al. 2017), junto con la gentrificación en zonas cercanas al lago Villarrica, invisibilización de la población mapuche y la creciente llegada de migrantes por estilo de vida (Guimond y Simard 2010; Zunino, Hidalgo y Marquardt 2011), entre otros cambios.

\section{Modelando los paisajes templados andinos}

Para modelar el paisaje del área de estudio, se utilizó un modelo de cobertura de suelo a partir de imágenes satelitales Landsat 7 ETM, cuya resolución espacial es de 30 × $30 \mathrm{~m}$. Se utilizó una 
técnica de clasificación temática mixta, la cual se basó en un modelo de cobertura de suelo desarrollado mediante el uso del software IDRISI Selva y una posterior actualización con elementos fragmentadores del paisaje, como los ejes viales. La validación del modelo fue realizada en un proceso mixto de trabajo en terreno y fotointerpretación de imágenes satelitales en color real.

Para el muestreo de los gradientes urbano-rurales, y con el propósito de verificar la existencia de una disminución en la densidad de caminos y en la densidad poblacional a medida que aumenta la distancia de la zona urbana, se generaron 14 transectos lineales dentro de la cuenca del lago Villarrica. Los transectos fueron dispuestos de manera aleatoria desde la ciudad de Pucón como centro urbano, ya que concentra la mayor densidad de actividades turísticas y presenta mayor cercanía a las áreas silvestres protegidas. Se tomó un total de 71 áreas de influencia circulares de $2 \mathrm{~km}$ de radio y situadas en los transectos a una distancia de $5 \mathrm{~km}$ (Figura 2). Se establecieron como parámetros un mínimo de tres microunidades de paisaje por transecto y la incorporación de microunidades situadas en el límite del área de estudio. La microunidad correspondiente al kilómetro 0 fue común para todos los transectos al situarse sobre la ciudad de Pucón.

Figura 2

Diseño muestral compuesto de 14 transectos lineales y 71 microunidades de paisaje



Fuente: Elaboración propia. 
Las circunferencias, en formato shapefile, fueron utilizadas para extraer microunidades de paisaje desde el modelo de cobertura de suelo en el software ArcGIS 10.4. A partir de estas microunidades de paisaje, se calculó:

a) Índice de Hemerobia (IH), como el grado de intervención determinado a partir de los porcentajes de territorio que ocupa cada uso o cobertura de suelo mediante la asignación de impactos correspondientes a los factores de Hemerobia y su asignación cualitativa (Cuadro 1).

$$
I H=\sum_{n=1}^{i n} \frac{f_{\pi}}{m} h
$$

donde, $\mathrm{m}$ : número de categorías de Hemerobia, $\mathrm{f}_{\mathrm{m}}$ : proporción del área de la categoría $\mathrm{m}$, h: factor de Hemerobia (lineal desde $h=1$ a h=m, Tabla 1). $\mathrm{IH}_{\text {máx }}=1$.

b) métricas de paisaje, como vía para la cuantificación de la composición y configuración de los parches de vegetación nativa. Los cálculos se realizaron en el software FRAGSTATS 4.2.1, caracterizando un total de 70 micro unidades. Para ello se utilizaron cinco métricas para la clase bosque y cinco métricas a escala de paisaje (Cuadro 2).

c) Sobre el centroide de las microunidades de paisaje, se extrajeron métricas básicas de topografía, tales como las medias de altitud y pendiente. Estos datos fueron calculados a partir del Modelo Digital de Elevación ASTER GDEM, cuya resolución espacial es de 30 × 30 m. Estas métricas se extrajeron con el software ArcGIS 10.4 mediante estadística zonal.

Cuadro 1

Tipo de uso o cobertura de suelo, de acuerdo al Grado de Hemerobia

\begin{tabular}{|c|c|l|}
\hline Factor & Grado de Hemerobia & \multicolumn{1}{c|}{ Uso o cobertura de suelo } \\
\hline $\mathbf{1}$ & Ahemerobia & Bosque nativo, glaciares y nieve \\
\hline $\mathbf{2}$ & Oligohemerobia & Renoval, cuerpos de agua y humedales \\
\hline $\mathbf{3}$ & Mesohemerobia & Matorral \\
\hline $\mathbf{4}$ & $\beta$-euhemerobia & Praderas y plantaciones forestales \\
\hline $\mathbf{5}$ & $\alpha$-euhemerobia & Agricultura y ganadería \\
\hline $\mathbf{6}$ & metahemerobia & Áreas urbanas e industriales \\
\hline
\end{tabular}

Fuente: modificado a partir de Steinhardt et al. (1999).

Se calculó el índice de correlación Tau-b de Kendall para determinar el grado de relación del IH con la distancia y la topografía, las métricas para la clase bosque y las métricas de paisaje en las microunidades de paisaje. La relación entre el nivel de intervención se determinó comparando los 
$r^{2}$ resultantes de un análisis de regresión simple entre cada una de las métricas y el IH. Los análisis se realizaron utilizando el software PASW 18.

Cuadro 2

Selección de métricas de paisaje a escala de clase (bosque) y paisaje para el análisis de correlación con el Índice de Hemerobia

\begin{tabular}{|l|l|l|}
\hline Métricas de paisaje & Abreviación & \multicolumn{1}{|c|}{ Descripción } \\
\hline Escala de clase (bosque) & PLAND & Porcentaje total de parches del mismo tipo \\
\hline Porcentaje de paisaje & NP & Número de parches del mismo tipo \\
\hline Número de parches & LPI & $\begin{array}{l}\text { Porcentaje que ocupa el parche más grande } \\
\text { dentro del paisaje }\end{array}$ \\
\hline Índice del parche más grande media & AREA_MN & $\begin{array}{l}\text { Promedio de la superficie de parches de un } \\
\text { mismo tipo en hectáreas }\end{array}$ \\
\hline Forma media & SHAPE_MN & $\begin{array}{l}\text { Aproximación de la forma de los parches } \\
\text { a una forma euclidiana simple (cuadrado o } \\
\text { círculo) }\end{array}$ \\
\hline Escala de paisaje & DIVISION & $\begin{array}{l}\text { Probabilidad de que dos puntos aleatorios } \\
\text { no se encuentren en parches del mismo tipo }\end{array}$ \\
\hline Índice de división del paisaje & SHDI & $\begin{array}{l}\text { Medida de diversidad del paisaje sensible a } \\
\text { tipos de parches poco frecuentes }\end{array}$ \\
\hline $\begin{array}{l}\text { Índice de diversidad de } \\
\text { Shannon }\end{array}$ & MSIDI & $\begin{array}{l}\text { Medida de diversidad del paisaje poco sen- } \\
\text { sible a tipos de parches poco frecuentes }\end{array}$ \\
\hline $\begin{array}{l}\text { Índice de diversidad } \\
\text { modificado de Simpson }\end{array}$ & SHEI & $\begin{array}{l}\text { Grado de uniformidad con que los tipos de } \\
\text { parches ocupan un paisaje }\end{array}$ \\
\hline $\begin{array}{l}\text { Índice de uniformidad de } \\
\text { Shannon }\end{array}$ & MSIEI & $\begin{array}{l}\text { Grado de uniformidad con que los tipos de } \\
\text { parches ocupan un paisaje }\end{array}$ \\
\hline $\begin{array}{l}\text { Índice de uniformidad } \\
\text { modificado de Simpson }\end{array}$ &
\end{tabular}

Fuente: modificado a partir de Mcgarigal et al. (2012), Pauchard et al. (2006) y Torres-Gómez et al. (2009).

Nuestra aproximación permitió realizar un análisis continuo; una perspectiva más cercana a la realidad y en función de procesos ecosistémicos entre las coberturas y no sólo dentro de las coberturas. De esta manera, los límites que se plantean en el levantamiento de unidades discretas (por ejemplo: un parche de bosque con límites definidos) se trabajaron de manera continua. Por otra parte, la propuesta metodológica supone sólo la revisión de un periodo, siendo de futuro interés revisar la historia evolutiva de los cambios en el gradiente urbano-rural a partir de intervenciones antrópicas (Arroyo-Rodríguez, Moreno y Galán-Acedo 2017). Una complejidad propia de la definición de unidades de paisaje es el tamaño a utilizar (Jackson y Fahrig 2012), siendo para este estudio una asignación en función de la influencia directa e indirecta de las dos áreas urbanas principales detectadas en la cuenca (Pucón y Villarrica). 


\section{Estructura del paisaje a partir de gradientes urbano- rurales}

Una de las cualidades principales del paisaje del bosque templado correspondió a la amplia extensión superficial de la clase bosque, a considerar como la agregación de bosque nativo adulto y renoval (Figura 3 y Cuadro 3). Los factores de Hemerobia de menor intervención antrópica 1 y 2 , que engloban a las clases de bosque junto a nieve, glaciares, humedales y cuerpos de agua, representaron el 72,72\% del paisaje de la cuenca del lago Villarrica (Tabla 2). Las categorías de intervención antrópica correspondientes a un uso intensivo se concentraron en un $12,51 \%$ de la superficie de la cuenca, equivalente a las plantaciones forestales, praderas y áreas urbanas e industriales. Un desglose más detallado señala la contribución individual de las clases, donde la extensión superficial del bosque nativo y el renoval equivalieron a un $59,3 \%$, mientras que el área urbana e industrial y plantaciones forestales equivalieron a un $2,21 \%$ del paisaje de la cuenca del lago Villarrica (Tabla 3). A partir del modelo de cobertura de suelo, no se detectó el grado de $\alpha$-euhemerobia correspondiente a agricultura y ganadería (Factor 5 ).

Figura 3

Disposición espacial del factor de Hemerobia en la cuenca del Lago Villarrica

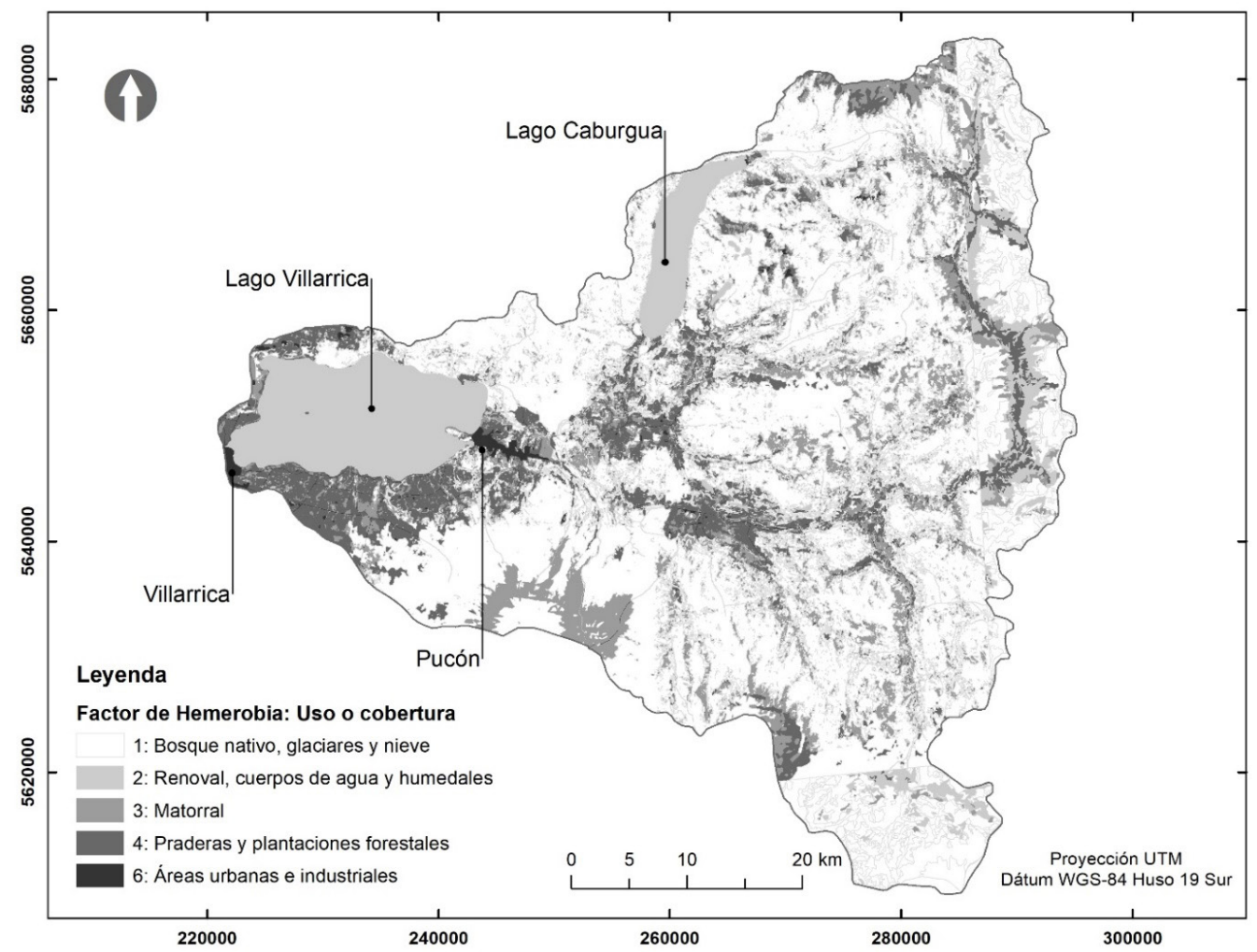

Fuente: Elaboración propia. 
Cuadro 3

Grado de Hemerobia en la cuenca del Lago Villarrica

\begin{tabular}{|c|l|r|}
\hline Factor & \multicolumn{1}{|c|}{ Uso o cobertura de suelo } & Porcentaje (\%) \\
\hline 1 & Bosque nativo, glaciares y nieve & 41,18 \\
\hline 2 & Renoval, cuerpos de agua y humedales & 31,55 \\
\hline 3 & Matorral & 14,77 \\
\hline 4 & Praderas, plantaciones forestales y silvicultura & 11,44 \\
\hline 6 & Áreas urbanas e industriales & 1,07 \\
\hline
\end{tabular}

Fuente: Elaboración propia.

Cuadro 4

Contribución porcentual por clase al paisaje de la cuenca del lago Villarrica

\begin{tabular}{|l|r|}
\hline Clases & \multicolumn{1}{|r|}{ Porcentaje (\%) } \\
\hline Bosque nativo & 36,74 \\
\hline Renoval & 22,56 \\
\hline Matorral & 14,77 \\
\hline Pradera & 10,30 \\
\hline Cuerpos de agua y humedales & 8,99 \\
\hline Suelo desnudo & 3,31 \\
\hline Plantaciones forestales & 1,14 \\
\hline Nieve y glaciares & 1,13 \\
\hline Área urbana & 1,07 \\
\hline
\end{tabular}

Fuente: Elaboración propia.

A partir del gradiente medio de antropización del paisaje, se distinguieron tres principales fenómenos (Figura 4). El primero correspondió a la variación del IH entre los 0 y $10 \mathrm{~km}$ desde el centro urbano, con un valor mínimo igual a 0,34. A partir de esto, se desprende tanto la acotación del área urbana como la tendencia de disminución en la antropización del paisaje al alejarse del punto central. El segundo fenómeno se representó en la inestabilidad del gradiente entre 10 y $45 \mathrm{~km}$, representando la discontinuidad espacial entre fragmentos de bosque nativo, renoval, matorral, praderas y plantaciones forestales. A $20 \mathrm{~km}$ del centro del área urbana se presentó una media de antropización mayor que en el entorno inmediato del área urbana condicionado por las extensiones de pradera, matorral y plantaciones forestales, coberturas de suelo asociadas a un uso extractivo o recreacional. El tercer fenómeno correspondió a la agrupación espacial a partir de los valores del IH, la desviación estándar del mismo y las métricas de diversidad/uniformidad en tres clases: 1) un área urbana consolidada entre 0 y $5 \mathrm{~km}$, 2) un área de uso intensivo acotado entre 10 y $20 \mathrm{~km}$, y con un máximo de intervención en los $20 \mathrm{~km}$, y 3) un área de conservación entre 25 y $45 \mathrm{~km}$, con un máximo de uniformidad en los $45 \mathrm{~km}$ al aumentar la clase bosque. 
El IH se encontró correlacionado con las métricas de Shannon, $\operatorname{SHDI}\left(r=0,51 ; r^{2}=0,26 ; P \leq 0,01\right)$ y SHEI $\left(r=0,47 ; r^{2}=0,22 ; P \leq 0,01\right)$, pero no así con las métricas de Simpson, MSIDI y MSIEI. A partir de la conceptualización de estas métricas, el paisaje de la cuenca del lago Villarrica presentó la presencia de parches poco comunes dentro de las microunidades de paisaje. Considerando la correlación a escala de clase del IH con la métrica de NP $\left(r=0,42 ; r^{2}=0,17 ; P \leq 0,01\right)$, se indica la presencia de parches de bosque nativo y de renoval en cercanías del centro urbano. Estos parches de bosque y renoval, a su vez, aumentan su tamaño y dominancia a medida a aumenta la distancia del centro urbano, calculado a partir de la correlación del IH con LPI $\left(r=-0,49 ; r^{2}=0,24 ; P\right.$ $\leq 0,01)$, AREA_MN $\left(r=-0,48 ; r^{2}=0,23 ; P \leq 0,01\right)$, y PLAND $\left(r=0,49 ; r^{2}=0,24 ; P \leq 0,01\right)$. No se detectó correlación entre IH y SHAPE_MN. Respecto a la influencia de la topografía, el IH disminuyó tanto por altitud $\left(r=-0,57 ; r^{2}=0,33 ; P \leq 0,01\right)$ como por pendiente $\left(r=-0,4 ; r^{2}=0,18 ; P \leq 0,01\right)$, denotando la disminución de la viabilidad de intervención antrópica y la concentración de coberturas y usos de suelo asociados a áreas urbanas, recreación y extracción de recursos en sectores de baja altitud y baja pendiente como aquellos cercanos al lago Villarrica.

Figura 4

Gradiente de antropización medio del paisaje de la cuenca del lago Villarrica

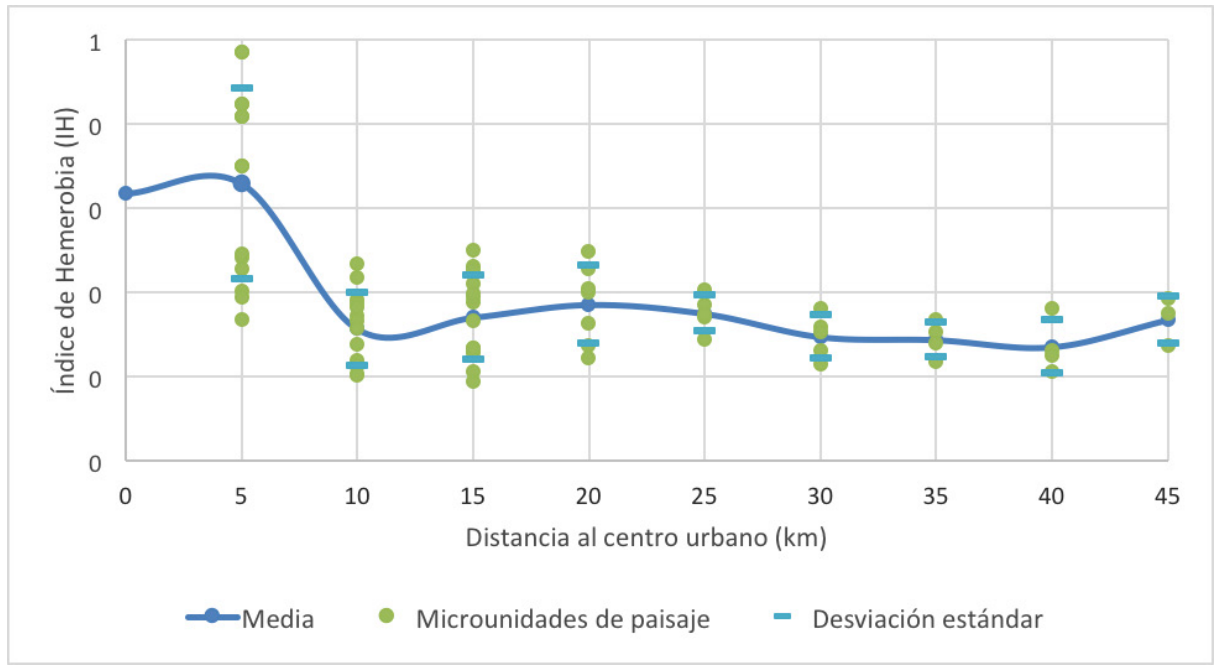

Fuente: Elaboración propia.

Debido al tamaño de la ciudad de Pucón, no existió una variación relevante respecto a los valores del IH entre el centroide de su área urbana y la media de las microunidades de paisaje correspondiente a los primeros $5 \mathrm{~km}(0,63$ a 0,66), condicionado además por la influencia del lago Villarrica de disminuir el grado de intervención. Sin embargo, debido a la localización y orientación de Pucón respecto al lago Villarrica, hay un aumento significativo de la microunidad del km $0(0,63)$ hacia el máximo valor del IH sobre intervención antrópica. Los transectos 3 y 4 hacia el este $(0,97), 14$ y 1 hacia el norte $(0,85), 7$ y 8 hacia el sur $(0,82)$ presentaron una gran relevancia al denotar las características fundamentales de un gradiente desde esta área urbana en direcciones 
donde no se encuentre el lago Villarrica y su influencia en el cálculo del índice a partir de la dominancia de la clase "cuerpos de agua".

La dominancia de clase se observó en los índices de diversidad/uniformidad (Figura 5), en los cuales hubo una disminución sustancial de la diversidad de clases en las microunidades hasta los $15 \mathrm{~km}$ desde el centro urbano, así como un posterior aumento hasta los $20 \mathrm{~km}$. Tanto por la distancia abarcada ( 0 a $45 \mathrm{~km}$ ), la similitud al gradiente medio de antropización y la baja dominancia de la clase agua, los transectos 3 y 4 señalaron las características generales de un ambiente con la capacidad de ser intervenido antrópicamente respecto a la variación de uso y cobertura de suelo. Las diferencias fueron observadas sobre la variación del gradiente de diversidad/uniformidad (Figura 6) pasando de un ambiente de alta diversidad de clases (cuerpos de agua, áreas urbanas e industriales, matorral, pastizal, humedales y plantaciones forestales) a un ambiente de baja diversidad y predominancia de una clase (bosque nativo) (Rocchini et al. 2012). Esto se encuentra asociado a la correlación del IH con la métrica DIVISION $\left(r=0,58 ; r^{2}=0,33 ; P \leq 0,01\right)$, donde la mayor diversidad implica que los parches presenten un bajo porcentaje del área total de la microunidad de paisaje y que la probabilidad de obtener aleatoriamente dos puntos de la misma clase sea menor. La disposición espacial de la clase bosque se encuentra, predominantemente, entre los 20 y $40 \mathrm{~km}$, marcando una fuerte diferencia sobre la influencia que presenta el lago Villarrica como cuerpo de agua dentro de la misma distancia (Figuras 5 y 6 ). El aumento final del gradiente al aumentar la distancia al centro urbano correspondió a la presencia de suelo desnudo y nieve, categorías presentes a partir del aumento de altitud y sobre los 1.600 m s.n.m., cercanos al límite de distribución altitudinal de Araucaria araucana (Vergara 2013).

Figura 5

Gradiente de diversidad/uniformidad medio del paisaje de la cuenca del lago Villarrica

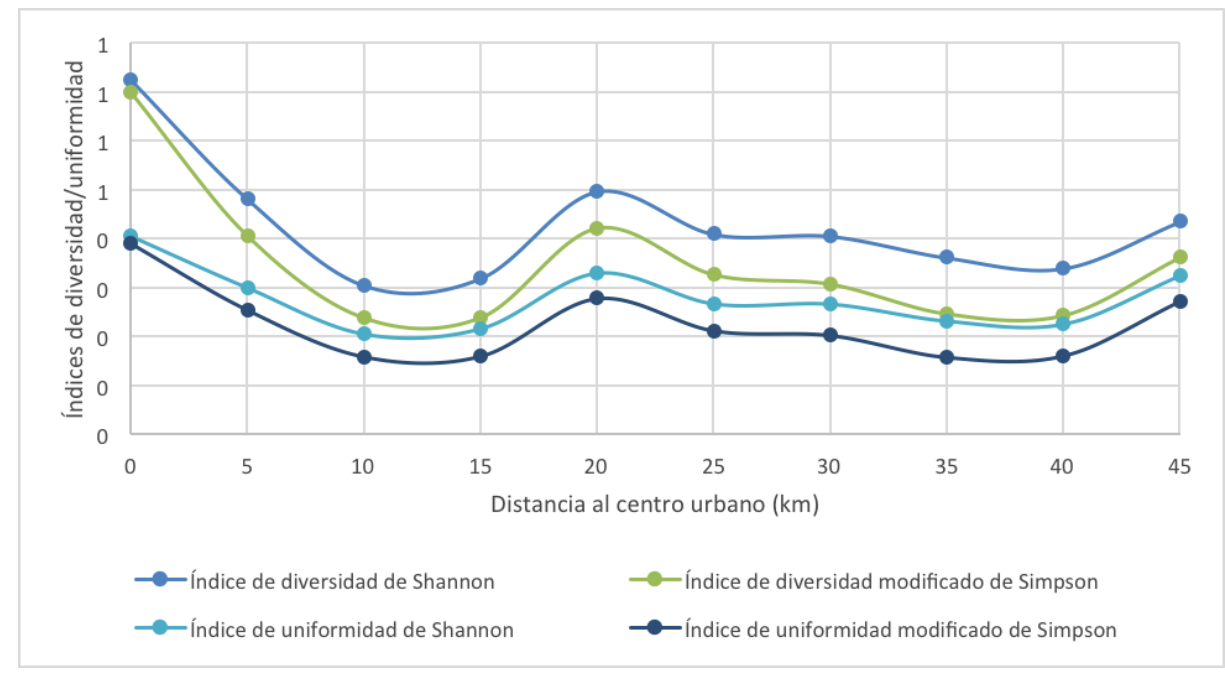

Fuente: Elaboración propia. 
Figura 6

Gradiente de diversidad/uniformidad medio para transectos 3 y 4 en la cuenca del lago Villarrica



Fuente: Elaboración propia.

\section{Oportunidades para la planificación territorial desde el concepto de paisaje}

La perspectiva de gradientes urbano-rurales permitió la aproximación desde metodologías con uso de datos discretos, como el cálculo del Índice de Hemerobia dentro de una microunidad de paisaje arbitraria, hacia el análisis de valores continuos en transectos. Esta aproximación permitió identificar procesos socio-ecológicos que trascienden la clasificación estricta de la cobertura o el uso de suelo. Por ejemplo, los resultados destacan las inflexiones en la perturbación antrópica a lo largo del paisaje que permiten establecer umbrales de áreas con cualidades propias, como el área de influencia del centro urbano.

El uso del concepto de gradiente supone una perspectiva al análisis de cambios donde es posible reconocer las intervenciones sobre los procesos de manera continua en el espacio. El análisis de gradientes urbano-rurales ha sido testeado y corroborado en múltiples países, tales como Estados Unidos (Nagy y Lockaby 2011; Warren et al. 2018; Tessel et al. 2014), México (Flesch y Steidl 2010) y Pakistán (Qureshi, Haase y Coles 2014). En todos los casos planteados, se ha observado la interacción de asignaciones discretas mediante índices o métricas en gradientes continuos, permitiendo presentar la relación espacial que sostienen, en conjunto a la distancia y la topografía, para analizar procesos dentro de una unidad de paisaje. Otros puntos de relevancia extraídos de la literatura son: a) la vinculación de patrones espaciales con procesos, principalmente en estudios socio-ecológicos o de contaminantes en áreas de intervención intensiva; b) la selección de recursos en respuesta a la variación de disponibilidad en el espacio; c) la posibilidad 
de realizar estudios multiescalares al presentar flexibilidad en la definición de microunidades de paisaje; d) la vinculación de cada subsector reconocido con estudios particulares según sean los procesos que ocurren en su interior $y ;$ e) la presentación de intervenciones tácitas desde el marco normativo, aquellas que permiten, limitan o prohíben acciones sobre coberturas de suelo.

Las limitaciones respecto al uso y cobertura de suelo se presentaron en dos perspectivas, a considerar desde el resultado de la modelación de cobertura de suelo y sobre el propio paisaje como sistema, vinculando las características topográficas, vegetacionales y normativas. Respecto a los resultados de la modelación, se infirió que el factor 5, o grado de -euhemerobia (agricultura y ganadería), no se encontró presente en la cuenca del lago Villarrica debido a dos alternativas: 1) la baja extensión superficial que actualmente es utilizada para el desarrollo de la agricultura o la ganadería, limitando las capacidades inherentes a la resolución espacial del modelo de cobertura suelo (Vergara 2013); y 2) la disminución de la superficie destinada a uso agrícola o ganadero a través de la reconversión de usos de suelo destinados a uso urbano e industrial y de plantación forestal (Petitpas et al. 2016). Respecto a las limitaciones propias del paisaje en el cambio de uso o cobertura de suelo, se consideraron: 1) la condicionalidad del crecimiento del área urbana y el uso antrópico a partir de la topografía (Colantoni et al. 2016), y 2) la implicancia normativa sobre la planificación territorial y la conservación desde el Sistema Nacional de Áreas Silvestres Protegidas del Estado (SNASPE) (Mardones 1999). Ambas restricciones sobre el paisaje fueron analizadas por Torres-Gómez et al. (2009), presentando la designación política y la topografía como impedimentos para el cambio de uso de suelo y la explotación de recursos, siendo coincidentes que los sectores de mayor altitud y pendiente se encuentren dentro del SNASPE.

Al tomar el Índice de Hemerobia como una medida aplicada a gradientes, este índice toma una alta dependencia sobre las características topográficas que condicionan la geometría de la cuenca y, a su vez, sobre la definición de la unidad de paisaje. Desde una mirada al diseño muestral, tanto el número como la localización de microunidades de paisaje se encontraron condicionadas por la geometría de la cuenca del lago Villarrica, particularmente la relación entre área y forma. Si bien esta relación se encuentra bien documentada en término de análisis de parches individuales o por clase de parches, aún sigue siendo un tema en discusión sobre la definición de los límites de un paisaje y las escalas de análisis que aplican sobre éste (Jackson y Fahrig 2012). A pesar de la disminución del número de muestras al aumentar la distancia desde el centro urbano, es posible considerar que la definición de gradiente observada en diversos estudios pueda ser la base para generalizar estos resultados. De tal manera, los valores del Índice de Hemerobia, en las microunidades más lejanas al área urbana y con un menor número de muestras, se encontrarían relacionados a valores similares en las cuencas limítrofes.

El grado de antropización del paisaje de la cuenca del lago Villarrica se encuentra íntimamente relacionado con la afluencia de turistas y la oferta de servicios turísticos, reflejado por la valoración de éste como paisaje estético. Gobster, Nassauer, Daniel y Fry (2007) proponen una asociación entre las acepciones de paisaje ecológico y paisaje estético, indicando que 1) el ser humano toma una relación estrecha donde pueda percibir y experimentar directamente su entorno, 2) las interacciones con el entorno pueden derivar en perturbaciones que puedan afectar al ser humano o el paisaje y 3) las características contextuales del entorno pueden variar entre individuos hasta definir lo que es denominado como belleza escénica. En la práctica, se observa en la ciudad de Pucón un uso turístico intensivo en periodo estival y una creciente expansión ur- 
bana por construcción de segunda residencia (Martínez y Terra 2012), mientras que, al alejarse del centro urbano, la valoración de las actividades toma una menor intervención antrópica y el uso del espacio se focaliza en las cualidades propias del espacio, como áreas privadas o destinadas a la conservación con uso turístico (Vergara 2013; Petitpas et al. 2016).

La denominada "transición del bosque" sugiere un proceso de recuperación de la cobertura de bosque por sobre otras coberturas de suelo que, históricamente, habían ejercido presiones sobre el bosque (Mather 1992; Mather y Needle 1998). Petitpas et al. (2016) presentan evidencias de recuperación del bosque nativo, una tendencia que no es característica de la región, ni del país. Esta investigación puede complementar el planteamiento de Petitpas et al. (2016), donde la transición del bosque provendría a partir de una relación en los procesos espaciales de intervención antrópica, explicado a partir de la disminución del Índice de Hemerobia al aumentar la distancia al centro urbano. Eventualmente, la transición del bosque podría ser relacionada al uso turístico como un factor explicativo, donde la predilección del turista hacia experiencias en ambientes de "mayor naturalidad" podría ser una intervención beneficiosa. Sin embargo, se requieren de mayores estudios que fundamenten la hipótesis de predilección turística de sitios asociados a la calidad escénica y un menor grado de intervención antrópica en esta área de estudio. Ante esto, Wood et al. (2013) desarrollaron un algoritmo de modelación específica que cumple la finalidad de evaluar la predilección del turista desde un enfoque espacial y usando datos de "social media". Este algoritmo fue utilizado por Keeler et al. (2015) para evaluar la predilección por cuerpos de agua de mayor valor estético, sentando un precedente para nuevas investigaciones.

\section{Consideraciones finales}

Los análisis provenientes de distintas áreas de la ciencia se vuelcan en un lenguaje de especificidad, legando la necesidad de unificar perspectivas. Si bien el concepto de "paisaje" tiene una amplia variedad de acepciones, éstas tienen como punto en común la visión del paisaje como un sistema de relaciones. A partir de la composición del paisaje a partir de elementos estructurales (de diversas tipologías, según la disciplina desde la cual se analice) y la funcionalidad inherente de las relaciones entre dichos elementos, se componen la auto-organización y las propiedades emergentes propias de un sistema (de la Barrera 2012). Reconociendo la variabilidad de elementos estructurales, funcionalidades, contextos y escalas, el análisis se encuentra dentro de una trama con una complejidad variable en base a las relaciones de agrupación, conectividad, retroalimentación y composición, entre otras. Desde este enfoque, el análisis interdisciplinario inherente en torno a los paisajes tiene un rol fundamental en la proposición y valoración de fenómenos relacionales, tales como la influencia de la estructura en el paisaje (Lausch et al. 2015), la ocurrencia de incendios forestales (Altamirano et al. 2013), la conectividad ecológica funcional (Hernández et al. 2015), hasta la construcción y la evolución del paisaje en el ciclo de intervención antrópica y adaptación natural (Di Giminiani y Fonck 2015).

Este estudio presentó la relación espacial continua entre la intervención antrópica y las características de un entorno relativamente poco intervenido, a partir de las características topográficas y de las áreas silvestres protegidas, ambos factores que impiden o limitan la intervención antrópica. Este continuo se presenta como una tensión continua entre los procesos socio-ecológicos de intervención y adaptación hacia y desde el entorno inmediato, sin contar con la nece- 
sidad de límites espaciales arbitrarios y el uso de valores discretos. Esta inclusión e interrelación de conceptos sobre paisaje constituyen una vía para la transferencia de conocimientos sobre procesos y patrones ecológicos a la sociedad. La incorporación de los procesos que ocurren dentro de un paisaje es fundamental para la toma de decisiones dentro de la planificación territorial (Colantoni et al. 2016; Vitt, Gulyás y Matzarakis 2015). De esta manera, se puede potenciar el desarrollo de la planificación territorial integrando, a la vez, los procesos socio-ecológicos que forman parte de las distintas acepciones del concepto "paisaje" a una escala local (Opdam et al. 2013). Las implicancias de la inclusión pragmática puede representar repercusiones positivas en los distintos subsistemas que conforman el paisaje, abordando más que las dinámicas sociales y ecológicas, sino también políticas y económicas.

\section{Agradecimientos}

Los autores agradecen a CONICYT/FONDAP (15110006), CONICYT/FONDECYT de Inicio (11160932), Fondos de Protección Ambiental (09-078-2010 y 9-I-009-12) del Ministerio del Medio Ambiente, The Peregrine Fund, The Rufford Small Grants for Nature Conservation (14397-2) y a "NETBIOAMERICAS" CONICYT/Apoyo a la Formación de Redes Internacionales entre Centros de Investigación (REDES150047). También se agradece a T. A. Altamirano, A. Vermehren, Corporación Nacional Forestal (CONAF-La Araucanía). J. Laker (Kodkod: Lugar de Encuentros), M. Venegas y R. Sanhueza (Guías-Cañe), R. Timmerman, M. Sabugal, C. Délano, Santuario Kawellucó y muchos otros propietarios que desinteresadamente nos permitieron trabajar en sus terrenos.

\section{Referencias bibliográficas}

ALTAMIRANO, A., SALAS, C., YAITUL, V. y SMITH-, C., 2013. Influencia de la heterogeneidad del paisaje en la ocurrencia de incendios forestales en Chile Central. Revista de Geografía Norte Grande, no. 55 , pp. 157-170.

ARROYO-RODRÍGUEZ, V., MORENO, C. y GALÁN-ACEDO, C., 2017. La ecología del paisaje en México: logros, desafíos y oportunidades en las ciencias biológicas. Revista Mexicana de Biodiversidad, vol. 88, pp. 42-51.

BALLANTYNE, M. y PICKERING, C.M., 2015. Recreational trails as a source of negative impacts on the persistence of keystone species and facilitation. Journal of Environmental Management, vol. 159, pp. 48-57.

CAVIEDES, J. y IBARRA, J.T., 2017. Influence of Anthropogenic Disturbances on Stand Structural Complexity in Andean Temperate Forests: Implications for Managing Key Habitat for Biodiversity. Plos One, vol. 12, no. 1, pp. 1-18.

COLANTONI, A., GRIGORIADIS, E., SATERIANO, A., VENANZONI, G. y SALVATI, L., 2016. Cities as selective land predators? A lesson on urban growth, deregulated planning and sprawl containment. Science of the Total Environment, vol. 545-546, pp. 329-339. 
COSTANTINI, M.L., ZACCARELLI, N., MANDRONE, S., ROSSI, D., CALIZZA, E. y ROSSI, L., 2012. NDVI spatial pattern and the potential fragility of mixed forested areas in volcanic lake watersheds. Forest Ecology and Management, vol. 285, pp. 133-141.

CUSHMAN, S., GUTZWEILER, K., EVANS, J. y MCGARIGAL, K., 2010. The gradient paradigm: a conceptual and analytical framework for landscape ecology. Spatial Complexity, Informatics, and Wildlife Conservation. S.I.: s.n., pp. 83-108.

DE LA BARRERA, F., 2012. La transformación del paisaje rural-urbano y su efecto sobre los servicios ecosistémicos en una microcuenca de Santiago (Chile). S.I.: Universitat de Barcelona.

DI GIMINIANI, P. y FONCK, M., 2015. El paisaje como proceso de vida: Experiencias de domesticación del bosque en el sur de Chile. Revista de Geografía Norte Grande, no. 61, pp. 7-24.

ESPINOSA, P., 2016. Segregación socio-espacial en una urbe turística de elite: el caso de Pucón, Chile. Revista Mad, no. 34, pp. 155-178.

FLESCH, A.D. y STEIDL, R.J., 2010. Importance of environmental and spatial gradients on patterns and consequences of resource selection. Ecological Applications, vol. 20, no. 4, pp. 1021-1039.

GEDDA, M., 2011. Araucanía Chile: patrimonio natural y cultural de sus territorios. Santiago: Pehuén.

GOBSTER, P., NASSAUER, J., DANIEL, T. y FRY, G., 2007. The shared landscape: What does aesthetics have to do with ecology? Landscape Ecology, vol. 22, no. 7, pp. 959-972.

GUIMOND, L. y SIMARD, M., 2010. Gentrification and neo-rural populations in the Québec countryside: Representations of various actors. Journal of Rural Studies, vol. 26, no. 4, pp. 449-464.

HERNÁNDEZ, A., MIRANDA, M., ARELLANO, E., SAURA, S. y OVALLE, C., 2015. Landscape dynamics and their effect on the functional connectivity of a Mediterranean landscape in Chile. Ecological Indicators, vol. 48, pp. 198-206.

IBARRA, J.T., GÁLVEZ, N., ALTAMIRANO, T., CAVIEDES, J., ROJAS, I., BONACIC, C. y MARTIN, K., 2017. Seasonal dynamics of avian guilds inside and outside core protected areas in an Andean Biosphere Reserve of southern Chile. Bird Study, vol. 64, no. 3, pp. 410-420.

IBARRA, J.T., GÁlVEZ, N., GIMONA, A., ALTAMIRANO, T., ROJAS, I., HESTER, A., LAKER, J. y BONACIC, C., 2012. Rufous-legged Owl (Strix rufipes) and Austral Pygmy Owl (Glaucidium nanum) stand use in a gradient of disrupted and old growth Andean temperate forests, Chile. Studies on Neotropical Fauna and Environment, vol. 47, no. 1, pp. 33-40.

IBARRA, J.T. y MARTIN, K., 2015. Biotic homogenization: Loss of avian functional richness and habitat specialists in disturbed Andean temperate forests. Biological Conservation, vol. 192, pp. 418-427. 
JACKSON, H.B. y FAHRIG, L., 2012. What size is a biologically relevant landscape? Landscape Ecology, vol. 27, no. 7, pp. 929-941.

KEELER, B.L., WOOD, S.A., POLASKY, S., KLING, C., FILSTRUP, C.T. y DOWNING, J.A., 2015. Recreational demand for clean water: evidence from geotagged photographs by visitors to lakes. Frontiers in Ecology and the Environment, vol. 13, no. 2, pp. 76-81.

LAUSCH, A., BLASCHKE, T., HAASE, D., HERZOG, F., SYRBE, R.-U., TISCHENDORF, L. y WALZ, U., 2015. Understanding and quantifying landscape structure - A review on relevant process characteristics, data models and landscape metrics. Ecological Modelling, vol. 295, pp. 31-41.

MAO, X. yan, MENG, J. jun y WANG, Q., 2014. Tourism and land transformation: A case study of the Li River Basin, Guilin, China. Journal of Mountain Science, vol. 11, no. 6, pp. 1606-1619.

MARDONES, G., 1999. La conservación de la naturaleza en la planificación del territorio: los desafíos para las áreas silvestres protegidas de Chile. Revista de Geografía Norte Grande, vol. 26, pp. 97-103.

MARTínEZ, P. y TERRA, N., 2012. Territorio y territorialidad en el turismo, el caso de Pucón, región de La Araucanía. Revista Lider, vol. 20, pp. 155-173.

MATHER, A., 1992. The forest transition. Area, vol. 24, no. 4, pp. 367-379.

MATHER, A.S. y NEEDLE, C.L., 1998. The forest transition: a theoretical basis. Area, vol. 30, no. 2, pp. 117-124.

MCDONNELL, M. y PICKETT, S., 1990. Ecosystem structure and function along gradients: an unexploited urban-rural opportunity for ecology. Ecology, vol. 71, no. 4, pp. 1232-1237.

MCGARIGAL, K., CUSHMAN, S. y ENE, E., 2012. FRAGSTATS v4: Spatial Pattern Analysis Program for Categorical and Continuous Maps. 2012. S.I.: s.n.

MEDLEY, K., PICKETT, S. y MCDONNELL, M., 1995. Forest-Landscape Structure along an Urban-To-Rural Gradient. 1995. S.I.: s.n.

NAGY, R. y LOCKABY, B., 2011. Urbanization in the Southeastern United States: Socioeconomic forces and ecological responses along an urban-rural gradient. Urban Ecosystems, vol. 14, no. 1, pp. 71-86.

OPDAM, P., NASSAUER, J.I., WANG, Z., ALBERT, C., BENTRUP, G., CASTELLA, J.C., MCALPINE, C., LIU, J., SHEPPARD, S. y SWAFFIELD, S., 2013. Science for action at the local landscape scale. Landscape Ecology, vol. 28, no. 8, pp. 1439-1445.

PAUCHARD, A., AGUAYO, M. y ALABACK, P., 2006. Cuantificando la fragmentación del paisaje: las métricas y sus significados ecológicos. En: A. GREZ, J. SIMONETTI y R. BUSTAMANTE (eds.), 
Biodiversidad en ambientes fragmentados de Chile: patrones y procesos a diferentes escalas. S.I.: s.n., pp. 41-67.

PETITPAS, R., IBARRA, J.T., MIRANDA, M. y BONACIC, C., 2016. Patterns Over a 24-Year Period Show an Increase in Native Vegetation Cover and Decreased Fragmentation in Andean Temperate Landscapes, Chile. Ciencia e investigación agraria, vol. 43, no. 3, pp. 5-5.

PICON, M.C., DE LA BARRERA, F. y RUIZ, F., 2016. Gradientes Urbano-Natural: Enfoque Ecológico-Territorial para la Planificación Urbana en Santiago, Chile. Segundo Congreso de Investigación Interdisciplinaria en Arquitectura, Diseño, Ciudad y Territorio. Santiago: Facultad de Arquitectura, Diseño y Estudios Urbanos, Pontificia Universidad Católica de Chile, y Facultad de Arquitectura y Urbanismo, Universidad de Chile, pp. 343-347.

QURESHI, S., HAASE, D. y COLES, R., 2014. The Theorized Urban Gradient (TUG) method-A conceptual framework for socio-ecological sampling in complex urban agglomerations. Ecological Indicators, vol. 36, pp. 100-110.

ROCCHINI, D., DELUCCHI, L., BACARO, G., CAVALLINI, P., FEILHAUER, H., FOODY, G., HE, K., NAGENDRA, H., PORTA, C., RICOTTA, C., SCHMIDTLEIN, S., SPANO, L.D., WEGMANN, M. y NETELER, M., 2012. Calculating landscape diversity with information-theory based indices: A GRASS GIS solution. Ecological Informatics, vol. 17, pp. 82-93.

SERRANO GINÉ, D., 2012. Consideraciones en torno al concepto de unidad de paisaje y sistematización de propuestas. Estudios Geográficos, vol. 73, no. 272, pp. 215-237.

STEINHARDT, U., HERZOG, F., LAUSCH, A., MÜLLER, E. y LEHMANN, S., 1999. Hemeroby index for landscape monitoring and evaluation. Environmental Indices - System Analysis Approach, pp. 237-254.

TENERELLI, P., DEMŠAR, U. y LUQUE, S., 2016. Crowdsourcing indicators for cultural ecosystem services: A geographically weighted approach for mountain landscapes. Ecological Indicators, vol. 64, pp. 237-248.

TESSEL, S., HILDERBRAND, R., SCHMIT, J., LOOKINGBILL, T. y SUAREZ-RUBIO, M., 2014. Assessing national park resource condition along an urban-rural gradient in and around Washington, DC, USA. Ecological Indicators, vol. 42, pp. 147-159.

TESSER, C., 2000. Algunas reflexiones sobre los significados del paisaje para la geografía. En: NULL, Revista de Geografía Norte Grande, vol. 27, pp. 19-26.

TORRES-GÓMEZ, M., DELGADO, L., MARÍN, V. y BUSTAMANTE, R., 2009. Estructura del paisaje a lo largo de gradientes urbano-rurales en la cuenca del río Aisén (Región de Aisén, Chile). Revista Chilena de Historia Natural, vol. 82, no. 1, pp. 73-82. 
VERGARA, G., 2013. Análisis estructural y funcional de la comuna de Pucón, Región de La Araucanía, Chile. S.I.: Pontificia Universidad Católica de Chile.

VILA, J., VARGA, D., LLAUSÀS, A. y RIBAS, A., 2006. Conceptos y métodos fundamentales en ecología del paisaje (landscape ecology). Una interpretación desde la geografía. Documents d'Analisis Geogràfica, vol. 48, pp. 151-166.

VITT, R., GULYÁS, Á. y MATZARAKIS, A., 2015. Temporal differences of urban-rural human biometeorological factors for planning and tourism in Szeged, Hungary. Advances in Meteorology, vol. 2015, pp. 1-8.

WALZ, U. y STEIN, C., 2014. Indicators of hemeroby for the monitoring of landscapes in Germany. Journal for Nature Conservation, vol. 22, pp. 279-289.

WARREN, R., REED, K., OLEJNIZCAK, M. y POTTS, D., 2018. Rural land use bifurcation in the urban-rural gradient. Urban Ecosystems, vol. 21, no. 3, pp. 577-583.

WOOD, S.A., GUERRY, A.D., SILVER, J.M. y LACAYO, M., 2013. Using social media to quantify nature-based tourism and recreation. Scientific Reports, vol. 3.

ZU-YONG, W., JU-QIN, S. y FU-HUA, S., 2013. Study on the Model Building for the Influence of the Water Environment on Urban Tourism Ecological Capacity. Journal of Applied Sciences, vol. 13, no. 10, pp. 1760-1766.

ZUNINO, H., HIDALGO, R. y MARQUARDT, E., 2011. Vivienda social y segregación espacial en la ciudad de Pucón, Chile: Entre el enclaustramiento y la integración con el hábitat turístico. Revista INVI, vol. 26, no. 71, pp. 15-55. 